\title{
Past, present, and future surgical management of malignant epithelial neoplasms of the lacrimal gland*
}

\author{
JOHN WARREN HENDERSON \\ From Louisiana State University Medical Centre, Louisiana, USA.
}

It is a once in a lifetime honour to receive an invitation to present the Mustardé lecture, and it gives me a feeling of great inner warmth to fulfil this role at the fourth annual meeting of your society.

Your first president, Mr John C Mustardé, is a ubiquitous man with an outgoing personality who has probably lectured in all principal regions of the world except, possibly, the South Pole. When I received the invitation I had some misgiving that I could find a suitable subject in your speciality that had not already been addressed by $\mathrm{Mr}$ Mustardé in his thorough, inimitable style. However, a search of his extensive bibliography did not reveal any publication on the subject of malignant lacrimal gland tumours.

\section{A treacherous neoplasm}

This family of neoplasms has bedeviled me throughout my career-long love affair with orbital tumours. In the coverage of the subject today I have selected the adenoid cystic carcinoma as the prototype of the group, and I will direct most of my remarks to the management of this treacherous neoplasm. It is the most frequent and most malignant member of the malignant neoplasms intrinsic in the lacrimal gland. If we can find a cure for adenoid cystic carcinoma, the management of the other malignant epithelial tumours of the lacrimal gland will be a lesser challenge.

I also fear adenoid cystic carcinoma of the lacrimal gland because I consider it the most evil of all primary neoplasms of the orbit. Personally, I would rather take my chances of survival with malignant melanoma of the orbit than my chances of survival with adenoid cystic carcinoma. I have never cured a patient with this particular neoplasm nor have I

* The Mustardé lecture: presented at the fourth annual meeting of the European Socicty of Ophthalmic, Plastic, and Reconstructive Surgery, 12 September 1985, Amsterdam, The Netherlands

Correspondence to Professor John W Henderson, M D, 7313 Capistrano Drive, Shreveport, Louisiana 71105, USA. personally observed a well documented cure among patients managed elsewhere.

The term, adenoid cystic carcinoma, came into general usage about 30 years ago, at which time it was separated histologically from other epithelial neoplasms of the lacrimal gland and given free standing status among this family of tumours. ${ }^{12}$ However, the surgical management of this tumour precedes by several years the first study of the neoplasm with the microscope.

\section{The past}

An early in-depth account of this neoplasm is that of Theodor Billroth. ${ }^{3}$ Billroth, aged 27 , was an assistant in B R K Langenbeck's surgical clinic in Berlin when he published his study on multiple recurrences of the tumour from the same patient. Using the solvents and fixatives of his era, he was intrigued by a system of glass-clear compartments and knobs in the residue of the tumour that he believed was the stroma of the tumour. These amorphous compartments were long and narrow, and from this he derived the name cylinder tumour. Billroth's observations were later confirmed when similar tumours were studied under the mircoscope 10 to 15 years later. Billroth's name for the tumour, cylindroma, persisted for almost 100 years.

Billroth's patient was a 22-year-old man who underwent seven operations in an interval of $2^{1 / 2}$ years. First, the tumour was removed. Soon the eye was enucleated because of recurrent tumour. Next several exenterations were performed. Finally, portions of the orbital bone were cauterised with a hot iron. However, the tumour stubbornly recurred. Throughout this ordeal the sedatives and analgesics of that era had little effect on the patient's pain. By the time of the last operation the tumour had extended into the ethmoid bone, the pterygopalatine fossa, and filled the adjacent maxillary antrum. In the few weeks before death the patient became insane, probably from intracranial extension of the tumour. 
All the salient clinical frustrations in the management of this tumour are found in Billroth's description of this pitiful patient in 1856, and these frustrations are equally applicable to the patients of the present time. Even so, the latter undergo fewer operations, control of pain has improved, general anaesthesia is available, and palliative radiotherapy permits longer survival, but the fatal result today is nearly as frequent as it was over a century ago.

\section{More radical surgery}

For almost the first hundred years cylindromas were managed in a fashion similar to that of Billroth's patient and many surgeons confirmed the frequency of bone invasion by the tumour. However, removal of diseased bone was attempted only in cases of recurrent tumour. In such cases the bone usually was nibbled away in stages over a period of several operations. A more radical removal of orbital bone that resulted in exposure of the dura was shunned because of the high risk of sepsis, meningitis, and death.

Reese and Jones in 1964 expressed the corollary that, if the invasion of bone by recurrent tumour is so frequent, then the initial surgery should be more radical. They proposed the removal of the lateral wall and rim of the orbit, combined with an exenteration, earlier in the course of the disease than had previously been attempted (Fig. 1). ${ }^{4}$ At the time of their report this surgical procedure had been used on five patients with lacrimal gland neoplasms. Two of these five cases were adenoid cystic carcinomas. In all five patients their combined bone removal-exenteration procedure was not performed until the malignant nature of the neoplasm had been established by study of fixed tissue specimens. In essence this operation was the second surgical procedure on the patient that followed, at some variable interval, the initial surgical biopsy.

In the 20 years since the report of Reese and Jones ${ }^{4}$ many, surgeons have used their operation, or some modification, to eradicate malignant neoplasms of the lacrimal gland. To learn the effectiveness of this operation for adenoid cystic carcinoma I searched the literature for well documented cases that had passed a 10-year disease-free interval from the time of initial surgery, but I found no reports of survivors of this type. Also I recently wrote to several dozen physicians whom I knew were interested in the survival of patients with adenoid cystic carcinoma, and physicians who had been in practice long enough to have personal knowledge of patients who had undergone the Reese-Jones type of operation for the disease. Those who submitted data in response to my inquiry are listed in Table 1 . From this mail survey I found only one case that seemed to meet the minimal criterion of a 10-year disease-free interval.

This survivor was a patient of Dr Robert Byers, of Houston, Texas. The patient, a 61-year-old woman, had a biopsy of an adenoid cystic carcinoma of the lacrimal gland in 1965, and has been followed up for a
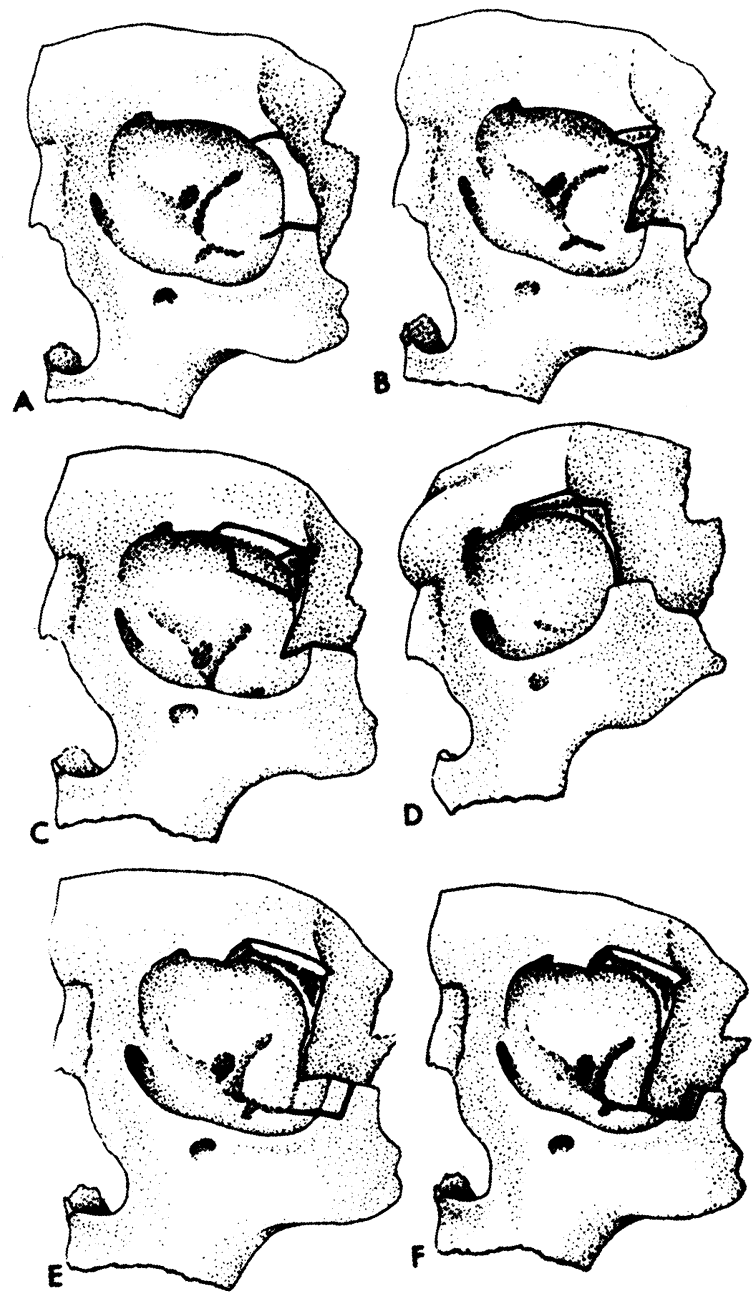

Fig. 1 Resection of orbital wall during exenteration. A: Bone resection is begun by cuts through lateral wall above and below with Stryker saw, as in Krönlein operation. B: Bone flap is broken posteriorly and removed and remaining broken edge is rongeured away as far posteriorly as possible. C: Bone resection is continued in area of lacrimal fossa by Stryker saw cut that is parallel to orbital rim and extends to mid point of superior rim. D: Broken posterior edge is removed with rongeurs as is necessary. E: Bone resection continued below by outlining adjacent inferior lateral wall with two Stryker saw cuts. F: Resection is completed by removing remaining bone as far as end of the inferior orbital fissure. (From Reese and Jones. ${ }^{4}$ ) 
Table 1 Physician participants in mail inquiry concerning survival of patients with adenoid cystic carcinoma of lacrimal gland

\begin{tabular}{llll}
\hline Name & City & Name & City \\
\hline Alper & Washington & Foos & Los Angeles \\
Bealc & Memphis & Jones & New York \\
Boniuk & Houston & Kennedy & Rochester NY \\
Bullock & Dayton & Kennerdell & Pittsburgh \\
Byers & Houston & McCord & Atlanta \\
Callahan & Birmingham & Reeh & Portland \\
Carroll & Minneapolis & Smith & New York \\
Char & San Francisco & Spencer & San Francisco \\
Cooper & New York & Waller & Rochester MN \\
Ellsworth & New York & Wilkins & Houston \\
\hline
\end{tabular}

period of seven years at the time of Byers and colleagues' publication." A short time after the biopsy procedure the neoplasm was removed by an orbital exenteration combined with removal of the lateral wall and rim of the orbit. The resected bone did not show gross evidence of tumour invasion and was not decalcified for histopatholical study, but the neoplasm had extended into the orbital connective tissue. Postoperatively the orbit was treated with radiotherapy.

This patient is living and well approximately 19 years (November 1984) from the time of the original surgery and has not had any local recurrence or metastasis of tumour in that interval (Byers R M, personal communication). The apparent absence of bone involvement by tumour may be one of the major factors in this patient's long survival.

\section{Almost always fatal}

Several respondents (Table 1) reported patients who were living 10 to 15 years after surgical diagnosis of their adenoid cystic carcinoma, but in each instance such patients had undergone one or more surgical procedures because of recurrent tumour, or were under therapy for metastasis. I believe such patients are still at risk of death from tumour.

From this type of survey I think it reasonable to assume that adenoid cystic carcinoma is almost always fatal. I believe a major factor in this dismal prognosis is that, over the years, we have underestimated the ability of this neoplasm to penetrate bone early in its growth. This penetration may be so insidious that it may be visible neither by radiography nor by gross inspection of bone. It is also my theory, but I have no clinical proof, that adenoid cystic carcinoma disseminates faster in bone than in soft tissue.

Once the tumour penetrates bone there are no tissue histiocytes or macrophages that might resist the spread of neoplasm along the marrow cavity of bone. In contrast the orbital soft tissues of some patients seem to resist the infiltration of tumour with a barrier of fibrosis and sclerosis. If tumour dissemination in bone is easier than tumour spread along the fascial planes of soft tissue, this is the reason why the limited bone removal of the Reese-Jones procedure may not cure the malignancy if bone invasion already is present at the time of the definitive surgery.

This point is illustrated by the course of a patient whose adenoid cystic carcinoma was removed by the combined Reese-Jones technique and followed up for a number of years. This patient was also treated with postoperative radiotherapy. (Jones IS, personal communication). The course of the patient in the eight years following surgery and radiotherapy was uneventful.' The exenterated orbit remained clean without sign of local recurrence of tumour. Suddenly, the patient developed fainting spells, loss of equilibrium, and died shortly thereafter. Necropsy revealed neoplasm in the brain adjacent to the treated orbit, probably due to direct extension of tumour that was present in bone beyond the boundary of the original surgical resection. The postoperative radiotherapy probably had had some suppressive effect on the multiplication of neoplastic cells in the eight-year interval of survival.

\section{Removal of bone}

If tumour dissemination through bone is a greater hazard than soft tissue infiltration in the ultimate course of the patient, it seems logical that bone should be removed at the time of the initial surgical procedure rather than some interval after either incisional or excisional biopsy. Accordingly a colleague and I proposed in 1976 an en bloc removal of the carcinoma and underlying bone as the initial surgical procedure.$^{6}$ Alas, in this patient histological study revealed extensive infiltration of tumour along the margin of the bone specimen, suggesting tumour dissemination beyond the area of bone resection. This patient ultimately died from metastasis.

Thirteen years ago Murray and colleagues also addressed the problem of removal of orbital bone in their publication on cranio-orbital resection. ${ }^{7}$ Other surgeons prior to this time had described radical removal of orbital bone for neoplasms secondarily invading the orbit from the sinuses, face, intracranial vault, and temple, but the Murray group was one of the first to target their procedure for primary orbital disease. Subsequently others have used the cranioorbital procedure for neoplasms of the orbit but have modified the operation both in the extent of bone removal and the method used to reconstruct the surgical defect. Basically all these operations remove more orbital bone and soft tissue in a monobloc than 
Fig. 2 Operative technique of regional cranio-orbital resection. 1: A circumorbital skin incision is made around the soft tissues and is carried down to periosteum. 2: The periosteum is elevated and multiple drill holes are made in frontal, nasal, malar, and maxillary bones. 3: The multiple burr holes are connected with the use of an osteotome. At this point the dura mater is separated from bone which encases the tumour. 4: Anatomical structure of the defect created by the surgical resection. Note that all structures between the frontal lobe and palate are expendable, once the eye has been removed. 5: The defect is resurfaced with split thickness skin graft. (From Murray et al. ${ }^{7}$ )

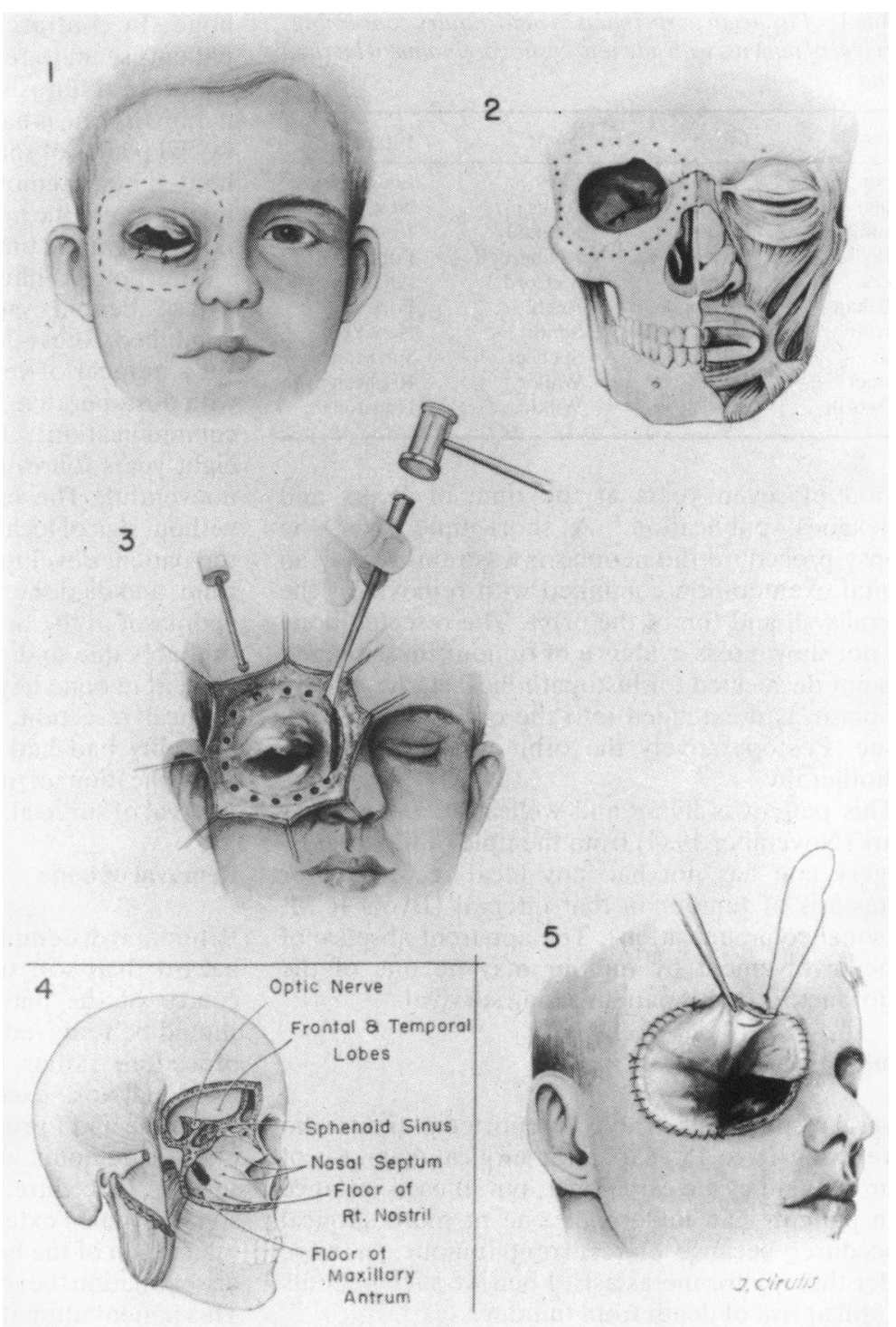

once thought possible, and the surgery is often performed by a team of surgeons from different surgical disciplines.

In the procedure of Murray et al. a circumorbital incision is made through skin down to periosteum (Fig. 2). Next, the periosteum is elevated. Multiple burr holes are drilled in the frontal, nasal, maxillary, and malar bones and the holes are connected with an osteotome. Then the bony box containing the tumour is separated from the adjacent dura. After severing the optic nerve anterior to the chiasm, all tissues from the frontal lobe to the palate are delivered as a monobloc. Monobloc resections of this general type are now used as the primary procedure for the removal of malignant neoplasm of the lacrimal gland in some tumour centres.

One such case of this type with a follow-up interval of seven years was reported by Wright in $1982 .{ }^{8}$ The patient, a 56-year-old man, underwent a monobloc removal of an adenoid cystic carcinoma of the lacrimal gland and the surrounding bone in March 1974. Histological study of the tissue specimen showed tumour extension into surrounding connective tissue, the lacrimal gland fascia, and the periosteum of the orbital roof, but there was no evidence of bone invasion (Wright $\mathrm{J} \mathrm{E}$, personal 
communication). Normal haemopoietic marrow was seen in one section of bone.

The patient was still tumour-free and in good health in September 1984, an interval of 10 years 5 months from the time of original surgery (Wright $J E$, personal communication). The role of the monobloc procedure in effecting this apparent ' 10 -year cure' would be more positive if there was histological evidence of tumour invasion of bone in the surgical specimen. If there was no marrow invasion by tumour in any of the bone not decalcified for histological study, an exenteration of the orbit might have sufficed for Wright's case.

Several respondents to my mail inquiry (Table 1) also mentioned a few additional cases of adenoid cystic carcinoma that had undergone the monobloc bone-tumour resection procedure, where tumour invasion of bone was confirmed by histological study of the tissue specimen. However, the tumour-free interval of these cases has not yet approached 10 years. At present I believe a tumour-free interval of 10 years is the minimum to permit cautious consideration of a cure of adenoid cystic carcinoma.

\section{The Future}

On the basis of the cases cited above there seems reasonable proof that exenteration of the orbit may cure a few cases of adenoid cystic carcinoma of the lacrimal gland provided the neoplasm has not infiltrated the soft tissue beyond the boundary of the dissection, has not invaded bone, and has not metastasised at the time of the definitive surgical excision. Another five to eight years must pass before we can judge the effectiveness of the newer monobloc, tumour-bone dissection procedure in those patients with known tumour invasion of bone.

The challenge of the future is, first, to detect early spread of tumour into orbital bone by techniques superior to the present ones of standard roentgenography, computed tomography, and gross inspection of bone. This would determine whether orbital bone resection, in addition to exenteration, is necessary to cure the patients of their neoplasm. Secondly, methods must be discovered to determine the extent of the tumour spread in bone. Such information would determine how much bone removal is needed to encompass the tumour dissemination.

Such discoveries might involve more sophisticated radiosotope scanning of bone than at present available, methods for biopsy of the marrow cavity of orbital bone, or refinements in the nuclear magnetic resonance imaging of orbital bone. The latter provides an image of the marrow of orbital bone heretofore not visualised by other technical methods.

Some of these discoveries or others, not yet foreseen, may be the subject of some future Mustardé lecture.

\section{References}

1 Foote FW Jr, Frazell EL. Tumors of the major salivary glands. In: Atlas of tumor pathology, Section 4, Fascicle 11. Washington DC: Armed Forces Institute of Pathology, 1954.

2 Forrest AW. Epithelial lacrimal gland tumors: pathology as a guide to prognosis. Ophthalmology (Rochester) 1954; 58: 848-66.

3 Billroth T. Untersuchungen über die Entwicklung der Blutgefasse. Berlin: Reimer, 1856: 55-69.

4 Reese AB, Jones IS. Bone resection in the excision of epithelial tumors of the lacrimal gland. Arch Ophthalmol 1964; 71: 382-5.

5 Byers RM, Berkely RG, Luna M, et al. Combined therapeutic approach to malignant lacrimal gland tumors. Am J Ophthalmol 1975; 79: 53-5.

6 Henderson JW, Neault RW. En bloc removal of intrinsic neoplasms of the lacrimal gland. Trans Am Opthalmol Soc 1976; 74: 133-43.

7 Murray JE, Matson DM, Habal MB, et al. Regional cranio-orbital resection for recurrent tumors with delayed reconstruction. Surg Gynecol Obstet 1972; 134: 437-47.

8 Wright JE. Factors affecting the survival of patients with lacrimal gland tumors. Can J Ophthalmol 1982; 17: 3-9. 